OPEN ACCESS

Edited by:

Yumin Li,

Lanzhou University, China

Reviewed by:

Duilio Pagano,

Mediterranean Institute for Transplantation and Highly Specialized

Therapies (ISMETT), Italy

Francesca Marcon

Policlinico Tor Vergata, Italy

${ }^{*}$ Correspondence:

Hong Zhao

zhaohong@cicams.ac.cn

Jianqiang Cai

caijianqiang188@sina.com

${ }^{\dagger}$ These authors have contributed equally to this work and share first authorship

Specialty section:

This article was submitted to Gastrointestinal Cancers: Gastric \& Esophageal Cancers,

a section of the journal

Frontiers in Oncology

Received: 12 October 2021 Accepted: 15 December 2021 Published: 07 January 2022

Citation:

Chen Q, Deng Y, Chen J, Zhao J, Bi X Zhou J, Li Z, Huang Z, Zhang $Y$, Chen X, Zhao H and Cai J (2022) Impact of Postoperative Infectious

Complications on Long-Term Outcomes for Patients Undergoing Simultaneous Resection for Colorectal Cancer Liver Metastases: A Propensity Score Matching Analysis.

Front. Oncol. 11:793653. doi: 10.3389/fonc.2021.793653

\section{Impact of Postoperative Infectious Complications on Long-Term Outcomes for Patients Undergoing Simultaneous Resection for Colorectal Cancer Liver Metastases: A Propensity Score Matching Analysis}

Qichen Chen ${ }^{\dagger}$, Yiqiao Deng ${ }^{\dagger}$, Jinghua Chen, Jianjun Zhao, Xinyu Bi, Jianguo Zhou, Zhiyu Li, Zhen Huang, Yefan Zhang, Xiao Chen, Hong Zhao* and Jianqiang Cai *

Department of Hepatobiliary Surgery, National Cancer Center/National Clinical Research Center for Cancer/Cancer Hospital, Chinese Academy of Medical Sciences and Peking Union Medical College, Beijing, China

Objective: To investigate the impact of postoperative infectious complications (POI) on the long-term outcomes of patients with colorectal cancer liver metastasis (CRLM) after simultaneous resection of colorectal cancer and liver metastases.

Methods: Four hundred seventy-nine CRLM patients receiving simultaneous resection between February 2010 and February 2018 at our hospital were enrolled. A 1:3 propensity score matching analysis (PSM) analysis was performed to balance covariates and avoid selection bias. After PSM, 90 patients were distributed to the POI group, and 233 patients were distributed to the no POI group. A log-rank test was performed to compare the progression-free survival (PFS) and overall survival (OS) data. A multivariate Cox regression model was employed to identify prognostic factors influencing OS and PFS. A value of two-sided $P<0.05$ was considered statistically significant.

Results: Compared to patients in the no POI group, patients in the POI group were more likely to have hepatic portal occlusion (78.9\% vs. $66.3 \%, P=0.021)$, operation time $\geq 325$ $\min (61.1 \%$ vs. $48.1 \%, P=0.026)$, and intraoperative blood loss $\geq 200 \mathrm{ml}(81.1 \%$ vs. $67.6 \%, P=0.012$ ). In multivariate analysis, intraoperative blood loss $\geq 200 \mathrm{ml}(\mathrm{OR}=2.057$, $95 \% \mathrm{Cl}: 1.165-3.634, P=0.013)$ was identified as the only independent risk factor for $\mathrm{POI}$. Patients with POI had a worse PFS ( $P<0.001$, median PFS: 7.5 vs. 12.7 months) and a worse OS ( $P=0.010$, median OS: 38.8 vs. 59.0 months) than those without POI. After 1:3 PSM analysis, no differences in clinicopathologic parameters were detected between the POI group and the no POI group. Patients with POI had a worse PFS $(P=0.013$, median PFS: 7.5 vs. 11.1 months) and a worse OS ( $P=0.020$, median OS: 38.8 vs. 59.0 months) than those without POI. Multivariate analysis showed that $\mathrm{POI}$ was an independent 
predictor for worse PFS (HR=1.410, 95\% Cl: 1.065-1.869, $P=0.017)$ and worse OS ( $\mathrm{HR}=1.682,95 \% \mathrm{Cl}: 1.113-2.544, P=0.014)$.

Conclusions: POI can significantly worsen the long-term outcomes of CRLM patients receiving simultaneous resection of colorectal cancer and liver metastases and should be considered to improve postoperative management and make better treatment decisions for these patients.

Keywords: colorectal cancer liver metastases, simultaneous resection, propensity score matched, long-term outcomes, postoperation infection

\section{INTRODUCTION}

Colorectal cancer (CRC) is the third most common malignant cancer and the second leading cause of cancer-related death in the world (1). The liver is the most common metastatic site, and more than $50 \%$ (2) of patients with colorectal cancer will develop liver metastases during their lifetimes. Liver metastases are often the cause of death for these colorectal cancer liver metastasis (CRLM) patients.

Surgery remains the only curative method for these patients. Traditionally, surgeons usually choose staged resection of the primary lesion and liver metastases in two separate operations (3). However, with the development of surgical technology, the improvement of the safety of hepatectomy (4) and the successful preoperative systematic treatment (5), simultaneous resection of the primary tumor and liver metastases in one operation has been increasing (6). Simultaneous resection has the advantages of reducing medical costs (6) and lowering the risk of other metastatic diseases in the interval of primary tumor resection and liver metastasis resection. In addition, several retrospective studies (7-9) revealed that patients receiving simultaneous resection could have comparable long-term outcomes to those receiving staged resection. Moreover, a recent randomized controlled trial (10) revealed that long-term outcomes tended to be improved in the simultaneous resection group compared with the staged resection group.

Although simultaneous resection has been advocated by clinicians because of the above advantages, the procedure is associated with increased postoperative complications (POCs) (11-14). Postoperative infectious complications (POI), as one of the major POCs, occur in 4-22 (15) percent of patients who undergo surgical resection for malignant solid tumous,and have been proven to have a negative impact on the long-term outcomes for patients with cancers (16-18), including colorectal cancer (19), oesophageal cancer (17) and gastric cancer (16). However, the impact of POI on CRLM patients is not fully understood, and there is a lack of evidence about how POI affects the long-term outcomes of patients receiving simultaneous resection of colorectal cancer and liver metastases. The present study aimed to explore the impact of POI on the long-term outcome of CRLM patients receiving simultaneous resection and identify the predictive factors of POI to help improve postoperative management of these patients.

\section{METHODS}

\section{Data Collection and Patient Population}

The study was approved by the Institutional Review Board of the Cancer Hospital, Chinese Academy of Medical Sciences. The inclusion criteria were as follows: (I) pathologically proven liver metastases of colorectal adenocarcinoma and (II) treatment with simultaneous resection of the primary tumor and liver metastases for curative purposes. Patients were excluded for incomplete follow-up data, lack of clinical data or the presence of other malignant tumors. Then, the clinical data of 479 CRLM patients admitted to the hospital between February 2010 and February 2018 were collected and analysed retrospectively.

Detailed information on demographics, clinicopathological characteristics, medical treatment and oncological results was reviewed. Diverse POCs, such as respiratory system infection, urinary system infection, digestive system infection, wound infection, and sepsis, were defined as POI (19). Patients were divided into two groups (POI, $n=90$; no POI, $n=389$ ). Of those POCs, minor complications were defined as Clavien-Dindo I-II, while major complications were defined as Clavien-Dindo III-V via the Clavien-Dindo classification system.

\section{Treatment}

Appropriate treatment strategies for CRLM patients were discussed by a multidisciplinary team (MDT) composed of surgeons, oncologists and radiologists. Preoperative chemotherapy regimens, mainly consisting of 5-fluorouracil/ capecitabine and oxaliplatin/irinotecan, with or without bevacizumab and cetuximab, were recommended to patients with any high-risk factors for recurrence (20). Liver resections were divided into major and minor resections. Resections of more than two segments were described as major resection and other resections were defined as minor resection (21). The surgical information mainly included surgical margin, extent of liver resection (major liver resection and minor liver resection), interoperative portal blockade, and concomitant RFA.

\section{Follow-Up and Outcomes}

Patients were followed up with regular clinical examinations after surgery: the first follow-up date was one month after surgery, then every 3 months for 5 years, and every 1 year thereafter. The interval from the date of surgery to death or the last follow-up was defined as overall survival (OS). The interval 
from the date of surgery to progression or the last follow-up was defined as progression-free survival (PFS).

\section{Statistical Analysis}

Continuous variables are presented as medians with interquartile ranges (IQRs), and the Mann-Whitney $U$ test was used for analysis. Chi-square or Fisher's exact tests were performed to analyse categorical variables. Multivariable logistic regression analysis was employed to explore the relationships between various variables and POI. Propensity score matching (PSM) was conducted to balance covariates and reduce the selection bias between the POI group and the no POI group. The present study used the Kaplan-Meier method to calculate PFS and OS. To statistically compare the PFS and OS data, the log-rank test was performed. A Cox regression model was employed to identify prognostic factors influencing OS and PFS, with results presented as hazard ratios (HRs) with 95\% confidence intervals (CIs). Variables with $P<0.10$ in univariable analysis were included in the multivariable analysis. A value of two-sided $P<0.05$ was considered statistically significant. All statistical analyses were performed using SPSS version 22 software (Armonk NY, USA) and R software (http://www.rproject.org).

\section{RESULTS}

\section{Clinicopathological Characteristics}

A total of 479 patients were enrolled, and most were male (65.1\%), with a median age of 59.0 years (IQR 52.0-65.0). Comorbidity was observed in $202(42.2 \%)$ patients. These patients had a median operation time of $325.0 \mathrm{~min}$ (IQR 260.0-415.0), and 50.5\% of them had an operation time of more than $325.0 \mathrm{~min}$. A median intraoperative blood loss of $200.0 \mathrm{ml}$ (IQR 100.0-400.0) was observed in these patients; and $70.1 \%$ of patients had an intraoperative blood loss of more than $200.0 \mathrm{ml}$. Primary tumors located in the colon were observed in $57.6 \%$ of the patients, while primary tumors located in the right hemicolon were observed in $20.5 \%$ of the patients. The median diameter of the largest lesion was 2.5 (IQR $1.5-4.0$ ) $\mathrm{cm}$, and $43.0 \%$ of the patients had a lesion of larger than $3.0 \mathrm{~cm}$. In addition, $57.0 \%$ of the patients had more than one liver metastasis, with a median of 2.0 liver metastases (IQR 1.0-4.0). Of these patients, the proportion of T3-T4 stage was $92.3 \%$, and the proportion of positive lymph node metastases was $73.1 \%$. Two hundred and sixty-seven patients $(55.7 \%)$ received preoperative chemotherapy, while forty-six patients $(9.6 \%)$ received concomitant RFA. One hundred eighty-four patients (38.4\%) had liver metastases with a bilobar distribution. The proportion of POCs was 48.4\% (232/ 479), while $20.7 \%$ (99/479) had major complications and $27.8 \%$ $(133 / 479)$ had minor complications. Ninety patients (90/479, $18.8 \%)$ had a POI. Overall, the mean length of hospital stay was 10.0 days (IQR: 9.0-13.0), and admission rate to ICU was 7.5\% (36/479). POCs rate was 48.43\% (232/479). Major complication occurred in $99(20.66 \%)$ patients while minor complication occurred in $133(27.76 \%)$ patients.
There were no significant differences between the POI group and the no POI group in age, sex, body mass index (BMI), comorbidities, preoperative carcinoembryonic antigen (CEA), metastasis diameter and number, tumor differentiation, tumor location, primary tumor $\mathrm{T}$ stage or positive lymph node metastases. Compared to patients without POI, patients with POI were more likely to have hepatic portal occlusion $(78.9 \%$ vs. $66.3 \%, P=0.021)$, operation time $\geq 325 \mathrm{~min}(61.1 \%$ vs. $48.1 \%$, $P=0.026)$, a longer hospital stay $(P<0.001$, mean 16.0 vs. 10.0 days) and intraoperative blood loss $\geq 200 \mathrm{ml}$ ( $81.1 \%$ vs. $67.6 \%$, $P=0.012$ ). Besides After 1:3 PSM, 90 patients were distributed to the POI group, and 233 patients were distributed to the no POI group. No differences in clinicopathologic parameters were detected between the two groups. The detailed clinicopathologic characteristics of the two groups of patients before and after matching are described in Tables $\mathbf{1}$ and 2, respectively.

\section{Predictors for POI}

Among the patients, the proportion of patients with POI was $18.8 \%(90 / 479)$. In the univariate analysis (Table 3 ), operation time $\geq 325 \mathrm{~min}(P=0.027)$, intraoperative blood loss $\geq 200 \mathrm{~mL}$ $(P=0.013)$ and hepatic portal occlusion $(P=0.022)$ were significantly associated with POI. In addition, extrahepatic metastases $(P=0.087)$ and major liver resection $(P=0.079)$ showed a tendency towards POI. A multivariable logistic regression analysis was performed to identify factors that were independently associated with POI. The above predictors $(P<0.10)$ were included in the multivariate analysis, and intraoperative blood loss $\geq 200 \mathrm{ml}(\mathrm{OR}=2.057,95 \% \mathrm{CI}: 1.165$ 3.634, $P=0.013$ ) was identified as an independent predictive factor of POI (Table 3).

\section{Impact of POI on Long-Term Outcomes Before PSM}

At the time of analysis, 171 patients (35.7\%) had died, and 333 patients (69.5\%) had experienced recurrence. The median PFS was 11.7 (95\% CI: 10.3-13.1) months, and the 1-year, 3-year and 5-year PFS rates were $48.8 \%, 26.0 \%$ and $24.7 \%$, respectively. The median OS was 58.3 (95\% CI: 45.4-71.1) months, and the 1-year, 3-year and 5 -year survival rates were $94.7 \%, 64.4 \%$ and $48.2 \%$, respectively. Compared to patients without POI, patients with POI had a worse PFS ( $P<0.001$, median PFS: 7.5 vs. 12.7 months) (Figure 1) and a worse OS ( $P=0.010$, median OS: 38.8 vs. 59.0 months) (Figure 2).

Univariate analysis showed that a diameter of liver metastases $\geq 3 \mathrm{~cm}$, multiple liver metastases, bilobar liver distribution, poor differentiation, positive lymph node metastases, extrahepatic metastases, concomitant RFA, non-R0 resection, major liver resection, hepatic portal occlusion, operation time $\geq 325 \mathrm{~min}$ and POI were relevant $(P<0.05)$ to a decreased PFS, while intraoperative blood loss $\geq 200 \mathrm{ml}$ had a tendency $(P<0.10)$ towards a decreased PFS. Six independent prognostic factors for PFS were identified in the multivariable analysis: positive lymph node metastases $(\mathrm{HR}=1.910,95 \% \mathrm{CI}$ : 1.453-2.511, $P<0.001)$, extrahepatic metastases ( $\mathrm{HR}=1.783,95 \%$ CI: $1.273-2.449, P=0.001)$, R0 resection $(\mathrm{HR}=0.643,95 \% \mathrm{CI}$ : $0.505-0.819, P<0.001)$, major liver resection $(\mathrm{HR}=1.525,95 \%$ 
TABLE 1 | Postoperative infectious complications in CRLM patients before PSM $(n=479)$.

\begin{tabular}{|c|c|c|c|c|}
\hline Item & Infection $(n=90)$ & Non-infection $(n=389)$ & $P$ & All patients $(n=479)$ \\
\hline Age $\geq 60$ years, $n(\%)$ & $44(48.9 \%)$ & $177(45.5 \%)$ & 0.561 & $221(46.1 \%)$ \\
\hline Male & $58(64.4 \%)$ & $254(65.3 \%)$ & 0.879 & $312(65.1 \%)$ \\
\hline $\mathrm{BMl} \geq 24 \mathrm{~kg} / \mathrm{m}^{2}, \mathrm{n}(\%)$ & $36(40.0 \%)$ & $191(49.1 \%)$ & 0.119 & 227 (47.4\%) \\
\hline Comorbidity, n (\%) & $41(45.6 \%)$ & $161(41.4 \%)$ & 0.471 & $202(42.2 \%)$ \\
\hline ASA score 3-4, n (\%) & $13(14.4 \%)$ & $45(11.6 \%)$ & 0.451 & $58(12.1 \%)$ \\
\hline Preoperative CEA $\geq 10$ ng/ml, n (\%) & 39 (43.3\%) & $184(47.3 \%)$ & 0.497 & $223(46.6 \%)$ \\
\hline Primary site in colon, $\mathrm{n}(\%)$ & $56(62.2 \%)$ & $220(56.6 \%)$ & 0.327 & $276(57.6 \%)$ \\
\hline Right hemicolon, n (\%) & $21(23.3 \%)$ & $77(19.8 \%)$ & 0.453 & $98(20.5 \%)$ \\
\hline Diameter of liver metastases $\geq 3 \mathrm{~cm}, \mathrm{n}(\%)$ & $45(50.0 \%)$ & $161(41.4 \%)$ & 0.137 & 206 (43.0\%) \\
\hline Multiple liver metastases, n (\%) & $53(58.9 \%)$ & 220 (56.6\%) & 0.687 & $273(57.0 \%)$ \\
\hline Bilobar liver distribution & $34(37.8 \%)$ & 150 (38.6\%) & 0.891 & $184(38.4 \%)$ \\
\hline Poor differentiation, n (\%) & $32(35.6 \%)$ & 125 (32.1\%) & 0.533 & $157(32.8 \%)$ \\
\hline T3-T4 stage, n (\%) & $83(92.2 \%)$ & 359 (92.3\%) & 0.983 & $442(92.3 \%)$ \\
\hline Positive lymph node metastasis, $\mathrm{n}(\%)$ & $63(70.0 \%)$ & $287(73.8 \%)$ & 0.466 & $350(73.1 \%)$ \\
\hline Extrahepatic metastases, n (\%) & $13(14.4 \%)$ & $33(8.5 \%)$ & 0.084 & $46(9.6 \%)$ \\
\hline Concomitant RFA, n (\%) & $11(12.2 \%)$ & 35 (9.0\%) & 0.349 & $46(9.6 \%)$ \\
\hline R0 resection, $\mathrm{n}(\%)$ & $60(66.7 \%)$ & $302(77.6 \%)$ & 0.029 & $362(75.6 \%)$ \\
\hline Major liver resection, n (\%) & $50(55.6 \%)$ & $176(45.2 \%)$ & 0.077 & $226(47.2 \%)$ \\
\hline Pretreatment chemotherapy, n (\%) & $54(60.0 \%)$ & $213(54.8 \%)$ & 0.367 & 267 (55.7\%) \\
\hline Hepatic portal occlusion, n (\%) & $71(78.9 \%)$ & $258(66.3 \%)$ & 0.021 & $329(68.7 \%)$ \\
\hline Operation time, min (median, IQR) & $370.00(298.75-450.00)$ & $320.00(252.00-405.00)$ & 0.002 & $325.00(260.00-415.00)$ \\
\hline Operation time $\geq 325 \mathrm{~min}, \mathrm{n}(\%)$ & $55(61.1 \%)$ & $187(48.1 \%)$ & 0.026 & $242(50.5 \%)$ \\
\hline Blood loss, ml (median, IQR) & $200.00(200.00-525.00)$ & $200.00(100.00-400.00)$ & 0.008 & $200.00(100.00-400.00)$ \\
\hline Blood loss $\geq 200 \mathrm{ml}, \mathrm{n}(\%)$ & $73(81.1 \%)$ & $263(67.6 \%)$ & 0.012 & $336(70.1 \%)$ \\
\hline Blood transfusion, $\mathrm{n}(\%)$ & $25(27.8 \%)$ & $88(22.6 \%)$ & 0.299 & $113(23.6 \%)$ \\
\hline The length of hospital stay, day (median, IQR) & $16.00(12.00-22.00)$ & $10.00(8.00-12.00)$ & $<0.001$ & $10.00(9.00-13.00)$ \\
\hline ICU rate, $\mathrm{n}(\%)$ & $9(10 \%)$ & $27(6.9 \%)$ & 0.321 & $36(7.5 \%)$ \\
\hline
\end{tabular}

TABLE 2 | Postoperative infectious complications in CRLM patients after PSM ( $n=323)$.

\begin{tabular}{|c|c|c|c|c|}
\hline Item & Infection $(n=90)$ & Non-infection $(n=233$ ) & $\boldsymbol{P}$ & All patients $(n=323)$ \\
\hline Age $\geq 60$ years, $n(\%)$ & 44 (48.9\%) & 99 (42.5\%) & 0.299 & $143(44.3 \%)$ \\
\hline Male & $58(64.4 \%)$ & 158 (67.8\%) & 0.564 & $216(66.9 \%)$ \\
\hline $\mathrm{BMI} \geq 24 \mathrm{~kg} / \mathrm{m}^{2}, \mathrm{n}(\%)$ & $36(40.0 \%)$ & 115 (49.4\%) & 0.131 & $151(46.7 \%)$ \\
\hline Comorbidity, n (\%) & $41(45.6 \%)$ & $101(43.3 \%)$ & 0.720 & 142 (44.0\%) \\
\hline ASA score 3-4, n (\%) & $13(14.4 \%)$ & 25 (10.7\%) & 0.353 & 38 (11.8\%) \\
\hline Preoperative CEA $\geq 10$ ng/ml, n (\%) & 39 (43.3\%) & 109 (46.8\%) & 0.577 & 148 (45.8\%) \\
\hline Primary site in colon, $\mathrm{n}(\%)$ & $56(62.2 \%)$ & $128(54.9 \%)$ & 0.236 & $184(57.0 \%)$ \\
\hline Right hemicolon, n (\%) & $21(23.3 \%)$ & $41(17.6 \%)$ & 0.241 & $62(19.2 \%)$ \\
\hline Diameter of liver metastases $\geq 3 \mathrm{~cm}, \mathrm{n}(\%)$ & $45(50.0 \%)$ & 107 (45.9\%) & 0.510 & $152(47.1 \%)$ \\
\hline Multiple liver metastases, n (\%) & $53(58.9 \%)$ & $140(60.1 \%)$ & 0.844 & $193(59.8 \%)$ \\
\hline Bilobar liver distribution & $34(37.8 \%)$ & $106(45.5 \%)$ & 0.210 & $140(43.3 \%)$ \\
\hline Poor differentiation, n (\%) & $32(35.6 \%)$ & $76(32.6 \%)$ & 0.616 & 108 (33.4\%) \\
\hline T3-T4 stage, n (\%) & $83(92.2 \%)$ & $222(95.3 \%)$ & 0.283 & 305 (94.4\%) \\
\hline Positive lymph node metastasis, n (\%) & $63(70.0 \%)$ & $172(73.8 \%)$ & 0.489 & 235 (72.8\%) \\
\hline Extrahepatic metastases, n (\%) & $13(14.4 \%)$ & $26(11.2 \%)$ & 0.416 & $39(12.1 \%)$ \\
\hline Concomitant RFA, n (\%) & $11(12.2 \%)$ & $19(8.2 \%)$ & 0.259 & 30 (9.3\%) \\
\hline R0 resection, n (\%) & $60(66.7 \%)$ & $166(71.2 \%)$ & 0.421 & $226(70.0 \%)$ \\
\hline Major liver resection, n (\%) & $50(55.6 \%)$ & $120(51.5 \%)$ & 0.513 & 170 (52.6\%) \\
\hline Pretreatment chemotherapy, n (\%) & $54(60.0 \%)$ & $129(55.4 \%)$ & 0.451 & $183(56.7 \%)$ \\
\hline Hepatic portal occlusion, n (\%) & 71 (78.9\%) & $183(78.5 \%)$ & 0.945 & $254(78.6 \%)$ \\
\hline Operation time $\geq 325 \mathrm{~min}, \mathrm{n}(\%)$ & $55(61.1 \%)$ & $136(58.4 \%)$ & 0.653 & $191(59.1 \%)$ \\
\hline Blood loss, ml (median, IQR) & 200.00 (200.00-525.00) & 200.00 (200.00-500.00) & 0.541 & $200.00(200.00-500.00)$ \\
\hline Blood loss $\geq 200 m l, n(\%)$ & $73(81.1 \%)$ & 183 (78.5\%) & 0.610 & 256 (79.3\%) \\
\hline Blood transfusion, n (\%) & $25(27.8 \%)$ & 65 (27.8\%) & 0.983 & $90(27.9 \%)$ \\
\hline
\end{tabular}

CI: $1.210-1.920, P<0.001)$, operation time $\geq 325 \mathrm{~min}(\mathrm{HR}=1.349$, 95\% CI: 1.072-1.697, $P=0.011)$, and POI $(\mathrm{HR}=1.451,95 \% \mathrm{CI}$ : 1.110-1.896, $P=0.006$ ) (Table 4).

Univariate analysis revealed that a diameter of liver metastases $\geq 3 \mathrm{~cm}$, multiple liver metastases, bilobar liver distribution, T3-T4 stage, positive lymph node metastases, concomitant RFA, non-R0 resection, major liver resection, hepatic portal occlusion, operation time $\geq 325 \mathrm{~min}$, blood transfusion, pretreatment chemotherapy and POI were associated $(P<0.05)$ with decreased OS. Multivariate analysis 
TABLE 3 | Prognostic factors for postoperative infectious complications in CRLM patients before PSM.

\begin{tabular}{|c|c|c|c|c|}
\hline Factor & \multicolumn{2}{|c|}{ Univariate analysis } & \multicolumn{2}{|c|}{ Multivariate analysis } \\
\hline Age $\geq 60$ years & 0.561 & $1.146(0.724-1.813)$ & & \\
\hline $\mathrm{BMI} \geq 24 \mathrm{~kg} / \mathrm{m}^{2}$ & 0.120 & $0.691(0.434-1.102)$ & & \\
\hline Comorbidity & 0.471 & $1.185(0.747-1.879)$ & & \\
\hline ASA score 3-4 & 0.452 & $1.291(0.664-2.509)$ & & \\
\hline Right hemicolon & 0.454 & $1.233(0.713-2.134)$ & & \\
\hline Diameter of liver metastases $\geq 3 \mathrm{~cm}$ & 0.138 & $1.416(0.894-2.243)$ & & \\
\hline Multiple liver metastases & 0.687 & $1.100(0.691-1.752)$ & & \\
\hline Bilobar liver distribution & 0.891 & $0.967(0.603-1.552)$ & & \\
\hline T3-T4 stage & 0.983 & $0.991(0.421-2.334)$ & & \\
\hline Positive lymph node metastasis & 0.467 & $0.829(0.501-1.373)$ & & \\
\hline Operation time $\geq 325 \mathrm{~min}$ & 0.027 & $1.697(1.063-2.711)$ & & \\
\hline Intraoperative blood loss $\geq 200 \mathrm{ml}$ & 0.013 & $2.057(1.165-3.634)$ & 0.013 & $2.057(1.165-3.634)$ \\
\hline Blood transfusion & 0.300 & $1.316(0.783-2.210)$ & & \\
\hline
\end{tabular}

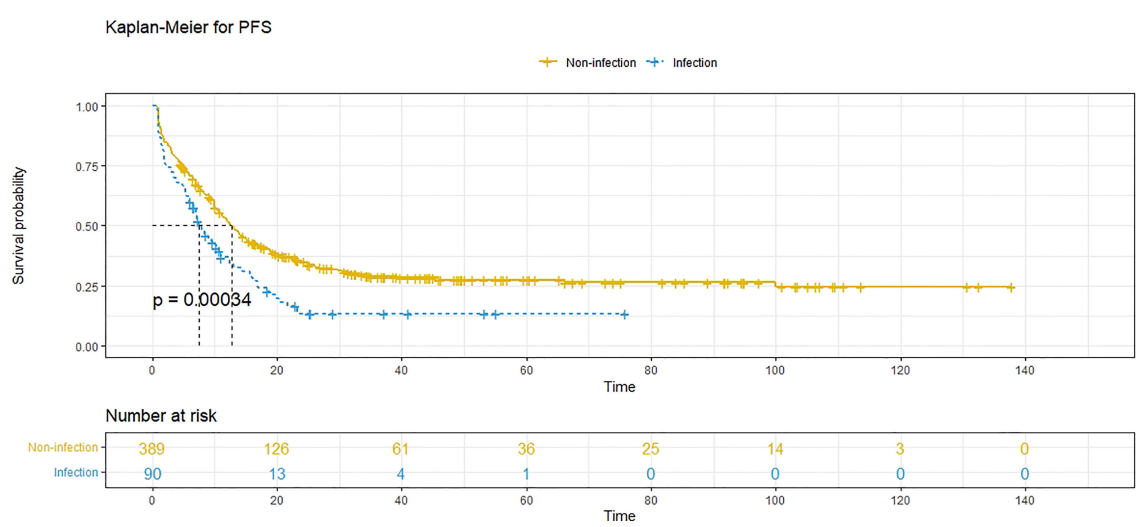

FIGURE 1 | Progression-free survival (PFS) comparison in patients with postoperative infective complications (blue line) and patients without postoperative infective complications (yellow line). Infection, postoperative infective complications. Non-infection, without postoperative infective complications.

revealed that $\mathrm{T} 3-\mathrm{T} 4$ stage $(\mathrm{HR}=3.228,95 \% \mathrm{CI}: 1.324-7.865$, $P=0.010)$, positive lymph node metastases $(\mathrm{HR}=2.676,95 \% \mathrm{CI}$ : $1.741-4.113, P<0.001)$, concomitant RFA ( $\mathrm{HR}=1.678,95 \% \mathrm{CI}$ : $1.109-2.538, P=0.014)$, major liver resection $(\mathrm{HR}=1.427,95 \%$ CI: $1.029-1.979, P=0.033$ ), and operation time $\geq 325 \mathrm{~min}$ $(\mathrm{HR}=1.735$, 95\% CI: 1.254-2.401, $P=0.001)$ were independent prognostic predictors of OS. However, POI ( $\mathrm{HR}=1.468,95 \% \mathrm{CI}$ : $1.000-2.155, P=0.050)$ was not independently associated with OS (Table 5).

\section{Impact of POI on Long-Term Outcomes After PSM}

A 1:3 PSM analysis was performed to balance covariates and avoid the selection bias of the retrospective study. Compared to patients without POI, patients with POI had a worse PFS ( $P=0.013$, median PFS: 7.5 vs. 11.1 months) (Figure 3) and a worse OS ( $P=0.020$, median OS: 38.8 vs. 59.0 months) (Figure 4). Multivariate analysis showed that POI was an independent predictor of both worse PFS ( $\mathrm{HR}=1.410,95 \% \mathrm{CI}: 1.065-1.869$, $P=0.017$ ) (Table 6) and worse OS (HR=1.682, 95\% CI: 1.113 2.544, $P=0.014$ ) (Table 7).

\section{DISCUSSION}

Cancer patients are susceptible to infections, and infection is a significant cause of death in this population (22). For colorectal cancer, bacterial infection (23-25) is of great significance to disease 


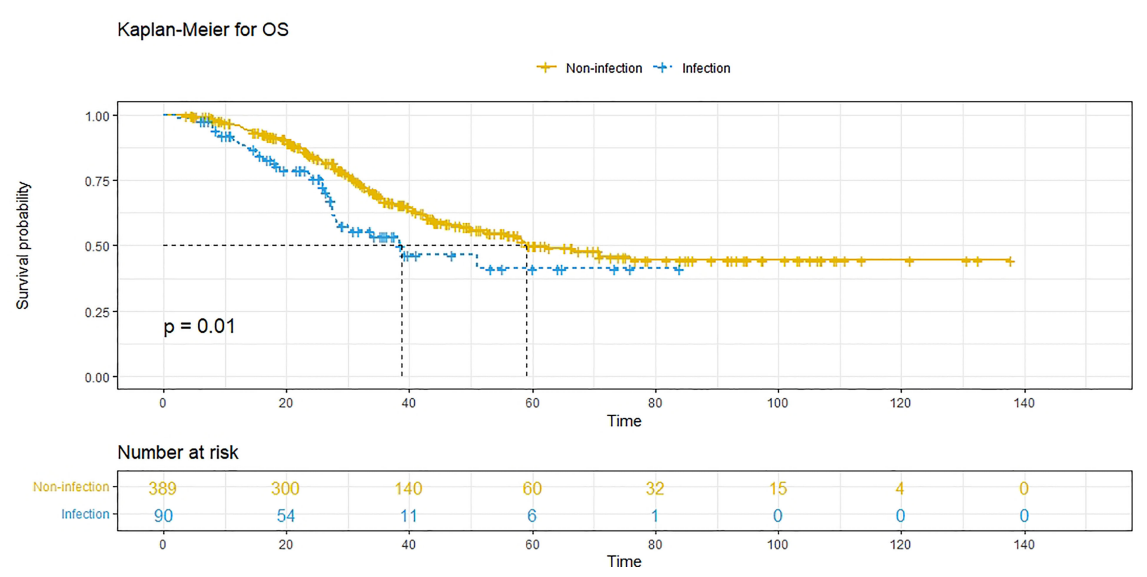

FIGURE 2 | Overall survival (OS) comparison in patients with postoperative infective complications (blue line) and patients without postoperative infective complications (yellow line). Infection, with postoperative infective complication. Non-infection, without postoperative infective complication.

TABLE 4 | Prognostic factors for PFS in CRLM patients before PSM.

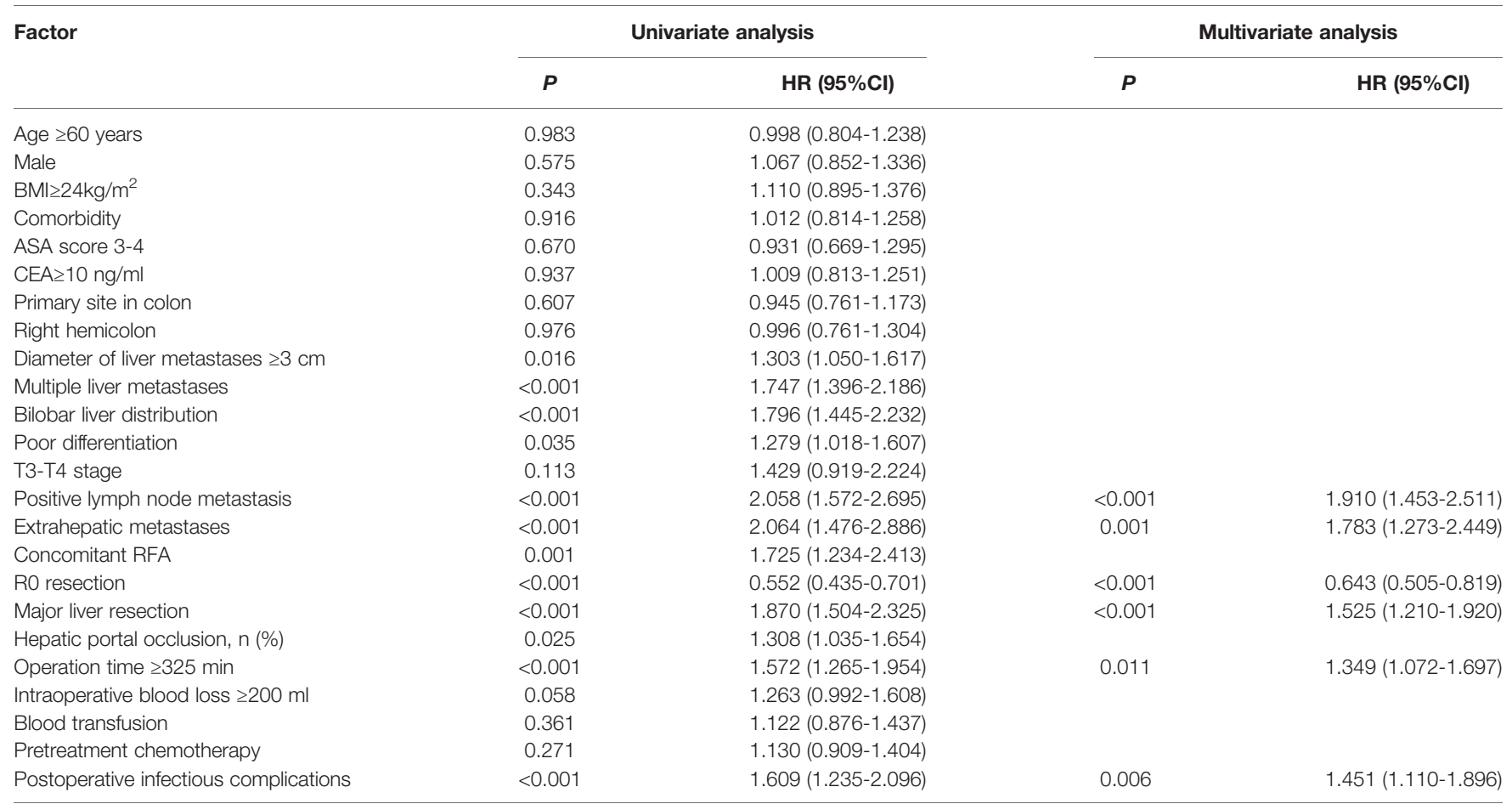

progression, such as metastases. Recently, a study suggested that infection caused by E. coli (26) could contribute to the formation of a protumorigenic environment in the liver and recruit circulating tumor cells, thus promoting liver metastases of colorectal cancer. All these findings indicate that the study of the relationship between infection and CRLM is important for clinical (27) purposes. The present study retrospectively analysed 479 CRLM patients receiving simultaneous resection of colorectal cancer and liver metastases, and PSM was performed to balance the effects of confounding factors. The results revealed that compared with patients in the no POI group, patients in the
POI group had a worse PFS and a worse OS before and after PSM. In addition, POI was an independent predictive factor for worse PFS and worse OS in multivariate analysis after PSM. To the best of our knowledge, this is the first study to examine the effect of POI on the long-term outcomes of CRLM patients receiving simultaneous resection of colorectal cancer and liver metastases. Significantly, it can help health care providers improve postoperative management and make better treatment decisions for these patients.

As one of the major POCs, POI remains a concern even in minimally invasive surgeries despite the development of modern 
TABLE 5 | Prognostic factors for OS in CRLM patients before PSM.

\begin{tabular}{|c|c|c|c|c|}
\hline Factor & \multicolumn{2}{|c|}{ Univariate analysis } & \multicolumn{2}{|c|}{ Multivariate analysis } \\
\hline Age $\geq 60$ years & 0.219 & $1.207(0.894-1.631)$ & & \\
\hline $\mathrm{BMI} \geq 24 \mathrm{~kg} / \mathrm{m}^{2}$ & 0.701 & $1.061(0.786-1.432)$ & & \\
\hline Comorbidity & 0.776 & $0.957(0.706-1.297)$ & & \\
\hline ASA score 3-4 & 0.403 & $0.816(0.506-1.315)$ & & \\
\hline Right hemicolon & 0.571 & $1.112(0.770-1.607)$ & & \\
\hline Diameter of liver metastases $\geq 3 \mathrm{~cm}$ & 0.030 & $1.396(1.033-1.887)$ & & \\
\hline Multiple liver metastases & 0.001 & $1.748(1.275-2.397)$ & & \\
\hline Bilobar liver distribution & 0.002 & $1.609(1.188-2.180)$ & & \\
\hline Poor differentiation & 0.110 & $1.305(0.942-1.808)$ & & \\
\hline T3-T4 stage & 0.010 & $3.221(1.323-7.843)$ & 0.010 & $3.228(1.324-7.865)$ \\
\hline Pretreatment chemotherapy & 0.006 & $1.550(1.136-2.116)$ & & \\
\hline Hepatic portal occlusion, n (\%) & 0.013 & $1.511(1.091-2.093)$ & & \\
\hline Operation time $\geq 325 \mathrm{~min}$ & $<0.001$ & $1.935(1.423-2.631)$ & 0.001 & $1.735(1.254-2.401)$ \\
\hline Intraoperative blood loss $\geq 200 \mathrm{ml}$ & 0.156 & $1.271(0.913-1.769)$ & & \\
\hline Blood transfusion & 0.014 & $1.515(1.087-2.111)$ & & \\
\hline Postoperative infectious complications & 0.011 & $1.635(1.120-2.388)$ & 0.050 & $1.468(1.000-2.155)$ \\
\hline
\end{tabular}

Kaplan-Meier for PFS

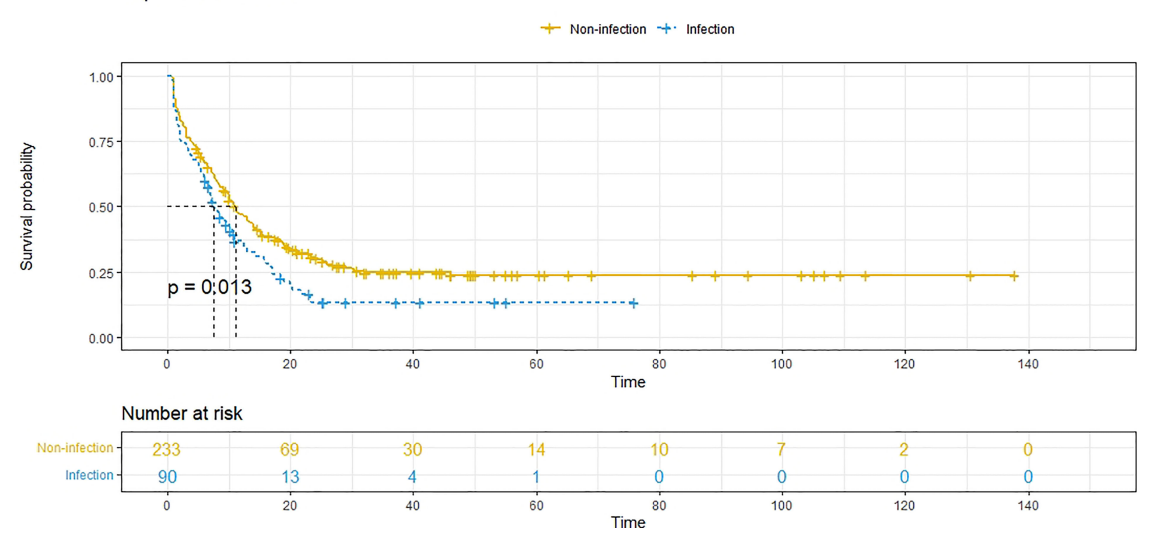

FIGURE 3 | Progression-free survival (PFS) comparison after propensity score matching in patients with postoperative infectious complications (blue line) and patients without postoperative complications (yellow line). Infection, with postoperative infective complication. Non-infection, without postoperative infective complication.

surgical technology. Generally, POI not only contributed to longer hospital stays and higher medical costs but was also associated with poor long-term outcomes of patients after surgery for various cancers. Nevertheless, only a few studies have focused on CRLM patients, and previous research is mainly limited to the effect of POI on CRLM patients who receive hepatectomy $(28,29)$. Regardless of the severity (28), POI was proven to be associated with decreased OS and PFS in CRLM patients who underwent hepatectomy. Compared with previous studies, the present study revealed for the first time that POI was significantly associated with worse PFS and OS in CRLM patients who underwent simultaneous resection.

With regard to the mechanism of the present study, the potential explanation can be postulated as follows: the explanations of previous studies mainly focused on the local or systemic inflammation caused by POI, which can suppress host immunity and promote the proliferation and migration of cancer cells or in the case of bacterial antigen-mediated processes (3032). Cancer cells can, at the early stage of infection, enhance their metastatic capability by activating Toll-like receptors (TLRs) and 


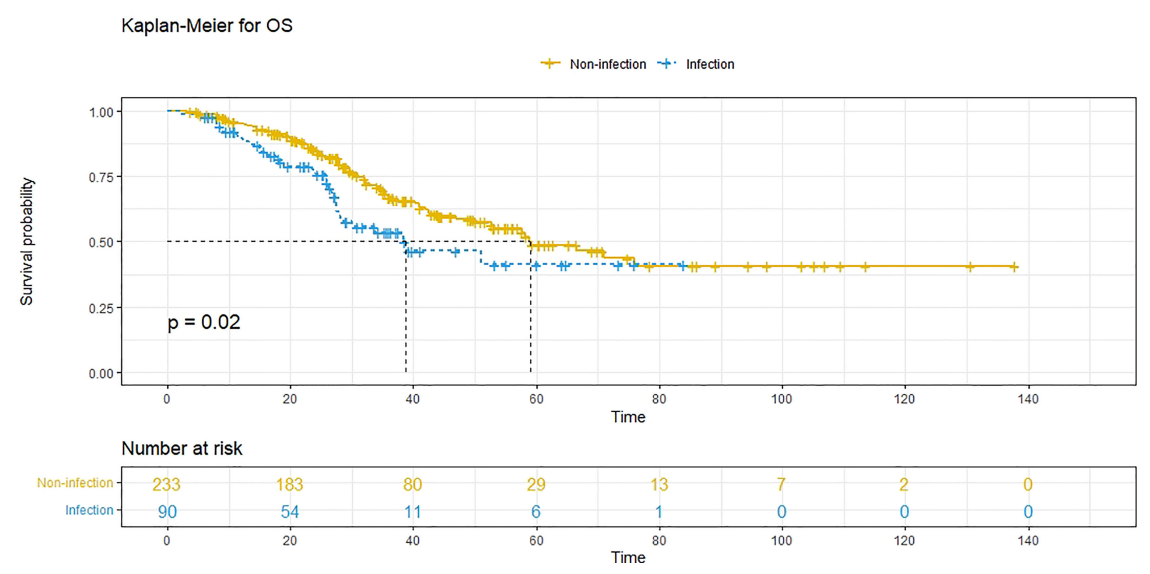

FIGURE 4 | Overall survival (OS) comparison after propensity score matching in patients with postoperative infectious complications (blue line) and patients without postoperative complications (yellow line). Infection, with postoperative infective complication. Non-infection, without postoperative infective complication.

TABLE 6 | Prognostic factors for PFS in CRLM patients after PSM.

\begin{tabular}{|c|c|c|c|c|}
\hline Factor & \multicolumn{2}{|c|}{ Univariate analysis } & \multicolumn{2}{|c|}{ Multivariate analysis } \\
\hline Age $\geq 60$ years & 0.783 & $0.965(0.746-1.247)$ & & \\
\hline $\mathrm{BMI} \geq 24 \mathrm{~kg} / \mathrm{m}^{2}$ & 0.366 & $1.125(0.872-1.451)$ & & \\
\hline Comorbidity & 0.848 & $0.975(0.754-1.261)$ & & \\
\hline ASA score 3-4 & 0.782 & $0.945(0.633-1.411)$ & & \\
\hline Right hemicolon & 0.716 & $1.061(0.771-1.460)$ & & \\
\hline Diameter of liver metastases $\geq 3 \mathrm{~cm}$ & 0.014 & $1.374(1.065-1.773)$ & & \\
\hline Multiple liver metastases & 0.002 & $1.534(1.176-2.002)$ & & \\
\hline Bilobar liver distribution & $<0.001$ & $1.575(1.220-2.032)$ & & \\
\hline Poor differentiation & 0.005 & $1.464(1.121-1.911)$ & & \\
\hline T3-T4 stage & 0.021 & $2.289(1.131-4.633)$ & 0.030 & $2.204(1.081-4.492)$ \\
\hline Pretreatment chemotherapy & 0.670 & $1.057(0.818-1.366)$ & & \\
\hline Hepatic portal occlusion, n (\%) & 0.539 & $1.100(0.812-1.491)$ & & \\
\hline Operation time $\geq 325 \mathrm{~min}$ & 0.026 & $1.345(1.035-1.747)$ & & \\
\hline Intraoperative blood loss $\geq 200 \mathrm{ml}$ & 0.027 & $1.459(1.045-2.036)$ & & \\
\hline Blood transfusion & 0.541 & $1.091(0.826-1.440)$ & & \\
\hline Postoperative infectious complications & 0.014 & $1.420(1.074-1.879)$ & 0.017 & $1.410(1.065-1.869)$ \\
\hline
\end{tabular}

creating a prometastatic environment throughout the body (33). Through systemic inflammation, POI can activate micrometastases (34), which cannot be detected by routine postoperative examination, to promote tumor recurrence or progression. More recently, Michela Perego (35) et al. found that stress-induced oxidized lipids can upregulate the fibroblast growth factor pathway in tumor cells, drive the reaction of dormant tumor cells and promote the development of new tumor lesions. Another potential explanation is the postponed postoperative chemotherapy $(36,37)$, which can be ascribed to
POI. The underlying mechanism remains to be fully elucidated, but the clinical utility of our study is still pronounced.

POI occurred in $18.8 \%$ (90/479) of patients in the present study, and the incidence was consistent with previous studies $(28,29)$ of patients who underwent abdominal surgeries. Because of its controllable and preventable nature, it is of great importance to identify the risk factors. In this study, the correlation of various characteristics and POI was analysed by regression analysis. Multivariate analysis indicated that intraoperative blood loss $\geq 200 \mathrm{ml}(P=0.013)$ was significantly 
TABLE 7 | Prognostic factors for OS in CRLM patients after PSM.

\begin{tabular}{|c|c|c|c|c|}
\hline \multirow[t]{2}{*}{ Factor } & \multicolumn{2}{|c|}{ Univariate analysis } & \multicolumn{2}{|c|}{ Multivariate analysis } \\
\hline & $\boldsymbol{P}$ & HR $(95 \% \mathrm{Cl})$ & $\boldsymbol{P}$ & HR $(95 \% \mathrm{Cl})$ \\
\hline Age $\geq 60$ years & 0.477 & $1.143(0.791-1.652)$ & & \\
\hline Male & 0.181 & $0.774(0.532-1.126)$ & & \\
\hline $\mathrm{BMl} \geq 24 \mathrm{~kg} / \mathrm{m}^{2}$ & 0.713 & $1.071(0.743-1.544)$ & & \\
\hline Comorbidity & 0.610 & $0.908(0.626-1.316)$ & & \\
\hline ASA score 3-4 & 0.967 & $1.012(0.568-1.804)$ & & \\
\hline $\mathrm{CEA} \geq 10 \mathrm{ng} / \mathrm{ml}$ & 0.417 & $1.164(0.807-1.678)$ & & \\
\hline Primary site in colon & 0.880 & $0.972(0.673-1.405)$ & & \\
\hline Right hemicolon & 0.439 & $1.188(0.767-1.840)$ & & \\
\hline Diameter of liver metastases $\geq 3 \mathrm{~cm}$ & 0.049 & $1.447(1.002-2.090)$ & & \\
\hline Multiple liver metastases & 0.006 & $1.739(1.171-2.582)$ & & \\
\hline Bilobar liver distribution & 0.028 & $1.511(1.046-2.182)$ & & \\
\hline Poor differentiation & 0.464 & $1.161(0.779-1.730)$ & & \\
\hline T3-T4 stage & 0.061 & $22.416(0.871-576.886)$ & & \\
\hline Positive lymph node metastasis & $<0.001$ & $2.963(1.721-5.101)$ & $<0.001$ & $3.002(1.736-5.190)$ \\
\hline Extrahepatic metastases & 0.604 & $1.160(0.662-2.033)$ & & \\
\hline Concomitant RFA & 0.004 & $2.060(1.258-3.372)$ & 0.058 & $1.643(0.983-2.749)$ \\
\hline Ro resection & 0.003 & 0.567 (0.390-0.824) & & \\
\hline Major liver resection & 0.001 & $1.867(1.282-2.719)$ & & \\
\hline Pretreatment chemotherapy & 0.003 & 1.780 (1.209-2.622) & 0.029 & $1.593(1.049-2.417)$ \\
\hline Hepatic portal occlusion, n (\%) & 0.085 & $1.466(0.949-2.264)$ & & \\
\hline Operation time $\geq 325 \mathrm{~min}$ & 0.002 & $1.816(1.234-2.673)$ & 0.029 & $1.587(1.049-2.402)$ \\
\hline Intraoperative blood loss $\geq 200 \mathrm{ml}$ & 0.168 & $1.396(0.869-2.243)$ & & \\
\hline Blood transfusion & 0.014 & $1.629(1.105-2.401)$ & & \\
\hline Postoperative infectious complications & 0.021 & $1.608(1.073-2.408)$ & 0.014 & $1.682(1.113-2.544)$ \\
\hline
\end{tabular}

correlated with POI. The possible explanations of POI caused by intraoperative blood loss are as follows: first, by increasing antiinflammatory cytokines and prostaglandins, intraoperative blood loss can cause cell-mediated immunosuppression (38); second, intraoperative blood loss can cause damage to the function of the intestinal barrier or break it, which would finally lead to invasion and dislocation of intestinal bacteria (39); third, through the alteration of the immune system, intraoperative blood loss can also increase the relative abundance of opportunistic pathogenic bacterial species in the intestinal tract (40). Given the relationship between intraoperative blood loss and POI, surgeons should take measures to reduce blood loss, such as identifying and managing patients at risk of high blood loss and improving techniques (carefully separating blood vessels, avoiding bleeding in a timely manner, hepatic portal occlusion et al.) to control bleeding during surgery (41). Surgeons can also improve perioperative care, such as maintaining the temperature in the operating room. Su SF et al. (42) found that heating, such as using a forced air heating system to maintain intraoperative normal temperature, can reduce the intraoperative blood loss of patients undergoing surgery. $\mathrm{Pu} \mathrm{Y}$ et al. (43) also found that the use of a bottom heating system can reduce intraoperative hypothermia in patients undergoing laparoscopic gastrointestinal surgery, thereby reducing intraoperative blood loss. Interestingly, Zei W. et al. (44) reported that gastric cancer patients who underwent neoadjuvant chemotherapy were associated with a higher incidence of POI. However, in the present study, neoadjuvant chemotherapy did not differ in the two groups. It's worth noting that the impact of neoadjuvant chemotherapy on POI is still controversial, many research reported that for local advanced colorectal cancer (45) and colorectal cancer with liver metastases
(46, 47), neoadjuvant chemotherapy would not increase the incidence of postoperative complications including POI. Besides, there were only 90 patients included and patients who underwent combined multiple organ resection were excluded in Zei W.'s study. While in the present study, all 479 CRLM patient underwent simultaneous resection of primary colorectal cancer and liver metastases. The heterogeneity of different cancer and the differences of surgical technique can definitely interfere the results.

There are several limitations in the present study. First, due to the retrospective and single-centre nature of this study, there could be some selection bias because of the lack of necessary randomized grouping. Second, the loss of the number of cases due to PSM may result in the loss of some patient information, and the probability of making the second type of error might increase. In the future, we will expand the sample size and look forward to conducting large prospective studies to further confirm our findings.

In conclusion, this is the first study on the impact of POI on the long-term outcomes of CRLM patients receiving simultaneous resection of colorectal cancer and liver metastases, which provides new decision-making evidence for clinicians to improve the preoperative, intraoperative and postoperative management of these patients. In the future, further application of these findings will help to improve the long-term outcomes of CRLM patients.

\section{DATA AVAILABILITY STATEMENT}

The raw data supporting the conclusions of this article will be made available by the authors, without undue reservation. 


\section{ETHICS STATEMENT}

The studies involving human participants were reviewed and approved by The Institutional Review Board of the Cancer Hospital, Chinese Academy of Medical Sciences. The patients/ participants provided their written informed consent to participate in this study.

\section{AUTHOR CONTRIBUTIONS}

Conception and design: HZ, JCa. Administrative support: HZ, JCa. Provision of study materials of patients: QC, YD. Collection

\section{REFERENCES}

1. Keum N, Giovannucci E. Global Burden of Colorectal Cancer: Emerging Trends, Risk Factors and Prevention Strategies. Nat Rev Gastroenterol Hepatol (2019) 16(12):713-32. doi: 10.1038/s41575-019-0189-8

2. Abdalla EK, Vauthey JN, Ellis LM, Ellis V, Pollock R, Broglio KR, et al. Recurrence and Outcomes Following Hepatic Resection, Radiofrequency Ablation, and Combined Resection/Ablation for Colorectal Liver Metastases. Ann Surg (2004) 239(6):818-25; discussion 825-7. doi: 10.1097/ 01.sla.0000128305.90650.71

3. Reddy SK, Barbas AS, Clary BM. Synchronous Colorectal Liver Metastases: Is it Time to Reconsider Traditional Paradigms of Management? Ann Surg Oncol (2009) 16(9):2395-410. doi: 10.1245/s10434-009-0372-1

4. Benson AB3rd, D'Angelica MI, Abbott DE, Abrams TA, Alberts SR, Saenz DA, et al. NCCN Guidelines Insights: Hepatobiliary Cancers, Version 1.2017. J Natl Compr Canc Netw (2017) 15(5):563-73. doi: 10.6004/jnccn.2017.0059

5. Julien LA, Thorson AG. Current Neoadjuvant Strategies in Rectal Cancer. J Surg Oncol (2010) 101(4):321-6. doi: 10.1002/jso.21480

6. Idrees JJ, Bagante F, Gani F, Rosinski BF, Chen Q, Merath K, et al. Population Level Outcomes and Costs of Single Stage Colon and Liver Resection Versus Conventional Two-Stage Approach for the Resection of Metastatic Colorectal Cancer. HPB (Oxford) (2019) 21(4):456-64. doi: 10.1016/j.hpb.2018.08.007

7. Abelson JS, Michelassi F, Sun T, Mao J, Milsom J, Samstein B, et al. Simultaneous Resection for Synchronous Colorectal Liver Metastases: The New Standard of Care? J Gastrointest Surg (2017) 21(6):975-82. doi: 10.1007/ s11605-017-3422-1

8. Hillingsø JG, Wille-Jørgensen P. Staged or Simultaneous Resection of Synchronous Liver Metastases From Colorectal Cancer-a Systematic Review. Colorectal Dis (2009) 11(1):3-10. doi: 10.1111/j.1463-1318.2008.01625.x

9. Chen J, Li Q, Wang C, Zhu H, Shi Y, Zhao G. Simultaneous vs. Staged Resection for Synchronous Colorectal Liver Metastases: A Metaanalysis. Int $J$ Colorectal Dis (2011) 26(2):191-9. doi: 10.1007/s00384-010-1018-2

10. Boudjema K, Locher C, Sabbagh C, Ortega-Deballon P, Heyd B, Bachellier P, et al. Simultaneous Versus Delayed Resection for Initially Resectable Synchronous Colorectal Cancer Liver Metastases: A Prospective, OpenLabel, Randomized, Controlled Trial. Ann Surg (2021) 273(1):49-56. doi: 10.1097/SLA.0000000000003848

11. Snyder RA, Hao S, Irish W, Zervos EE, Tuttle-Newhall JE, Parikh AA. ThirtyDay Morbidity After Simultaneous Resection of Colorectal Cancer and Colorectal Liver Metastases: American College of Surgeons NSQIP Analysis. $J$ Am Coll Surg (2020) 230(4):617-27.e9. doi: 10.1016/j.jamcollsurg.2019.12.018

12. Ono Y, Saiura A, Arita J, Takahashi Y, Takahashi M, Inoue Y. Short-Term Outcomes After Simultaneous Colorectal and Major Hepatic Resection for Synchronous Colorectal Liver Metastases. Dig Surg (2017) 34(6):447-54. doi: 10.1159/000455295

13. Bogach J, Wang J, Griffiths C, Parpia S, Saskin R, Hallet J, et al. Simultaneous Versus Staged Resection for Synchronous Colorectal Liver Metastases: A Population-Based Cohort Study. Int J Surg (2020) 74:68-75. doi: 10.1016/ j.ijsu.2019.12.009 and assembly of data: All authors. Data analysis and interpretation: QC, YD. Manuscript writing: All authors. Final approval of manuscript: All authors.

\section{FUNDING}

This study was supported by the National Natural Science Foundation of China (81972311, 82002611), the CAMS Innovation Fund for Medical Sciences (CIFMS) (Grant No. 201712M-4-002), the Non-profit Central Research Institution Fund of Chinese Academy of Medical Sciences (2019PT310026) and Sanming Project of Medicine in Shenzhen (No. SZSM202011010).

14. Pagano D, Gruttadauria S. A Commentary on "Simultaneous Versus Staged Resection for Synchronous Colorectal Liver Metastases: A Population-Based Cohort Study". Importance of Avoiding Any Other Additional Risk in Selected Patients With Synchronous Colorectal Liver Metastases. Int J Surg (2020) 74:111-2. doi: 10.1016/j.ijsu.2020.01.006

15. Buzquurz F, Bojesen RD, Grube C, Madsen MT, Gögenur I. Impact of Oral Preoperative and Perioperative Immunonutrition on Postoperative Infection and Mortality in Patients Undergoing Cancer Surgery: Systematic Review and Meta-Analysis With Trial Sequential Analysis. BJS Open (2020) 4(5):764-75. doi: 10.1002/bjs5.50314

16. Han WH, Oh YJ, Eom BW, Yoon HM, Kim YW, Ryu KW. Prognostic Impact of Infectious Complications After Curative Gastric Cancer Surgery. Eur J Surg Oncol (2020) 46(7):1233-8. doi: 10.1016/j.ejso.2020.04.032

17. Yamashita K, Makino T, Miyata H, Miyazaki Y, Takahashi T, Kurokawa Y, et al. Postoperative Infectious Complications are Associated With Adverse Oncologic Outcomes in Esophageal Cancer Patients Undergoing Preoperative Chemotherapy. Ann Surg Oncol (2016) 23(6):2106-14. doi: 10.1245/s10434015-5045-7

18. Yang T, Liu K, Liu CF, Zhong Q, Zhang J, Yu JJ, et al. Impact of Postoperative Infective Complications on Long-Term Survival After Liver Resection for Hepatocellular Carcinoma. Br J Surg (2019) 106(9):1228-36. doi: 10.1002/ bjs.11231

19. Artinyan A, Orcutt ST, Anaya DA, Richardson P, Chen GJ, Berger DH. Infectious Postoperative Complications Decrease Long-Term Survival in Patients Undergoing Curative Surgery for Colorectal Cancer: A Study of 12,075 Patients. Ann Surg (2015) 261(3):497-505. doi: 10.1097/SLA. 0000000000000854

20. Diagnosis And Treatment Guidelines For Colorectal Cancer Working Group CSOCOC. Chinese Society of Clinical Oncology (CSCO) Diagnosis and Treatment Guidelines for Colorectal Cancer 2018 (English Version). Chin J Cancer Res (2019) 31(1):117-34. doi: 10.21147/j.issn.1000-9604.2019.01.07

21. Thompson HH, Tompkins RK, Longmire WPJr. Major Hepatic Resection. A 25-year experience. Ann Surg (1983) 197(4):375-88. doi: 10.1097/00000658198304000-00001

22. Chen W, Zheng R, Baade PD, Zhang S, Zeng H, Bray F, et al. Cancer Statistics in China, 2015. CA Cancer J Clin (2016) 66(2):115-32. doi: 10.3322/ caac. 21338

23. Wassenaar TM. E. Coli and Colorectal Cancer: A Complex Relationship That Deserves a Critical Mindset. Crit Rev Microbiol (2018) 44(5):619-32. doi: 10.1080/1040841X.2018.1481013

24. Brennan CA, Garrett WS. Fusobacterium Nucleatum - Symbiont, Opportunist and Oncobacterium. Nat Rev Microbiol (2019) 17(3):156-66. doi: 10.1038/s41579-018-0129-6

25. Dejea CM, Fathi P, Craig JM, Boleij A, Taddese R, Geis AL, et al. Patients With Familial Adenomatous Polyposis Harbor Colonic Biofilms Containing Tumorigenic Bacteria. Science (2018) 359(6375):592-7. doi: 10.1126/ science.aah3648

26. Bertocchi A, Carloni S, Ravenda PS, Bertalot G, Spadoni I, Lo Cascio A, et al. Gut Vascular Barrier Impairment Leads to Intestinal Bacteria Dissemination 
and Colorectal Cancer Metastasis to Liver. Cancer Cell (2021) 39(5):70824.e11. doi: 10.1016/j.ccell.2021.03.004

27. Wong SH, Yu J. Gut Microbiota in Colorectal Cancer: Mechanisms of Action and Clinical Applications. Nat Rev Gastroenterol Hepatol (2019) 16(11):690704. doi: 10.1038/s41575-019-0209-8

28. Fernández-Moreno MC, Dorcaratto D, Garcés-Albir M, Muñoz E, Arvizu R, Ortega J, et al. Impact of Type and Severity of Postoperative Complications on Long-Term Outcomes After Colorectal Liver Metastases Resection. J Surg Oncol (2020) 122(2):212-25. doi: 10.1002/jso.25946

29. Memeo R, de Blasi V, Adam R, Goéré D, Laurent A, de'Angelis N, et al. Postoperative Infectious Complications Impact Long-Term Survival in Patients Who Underwent Hepatectomies for Colorectal Liver Metastases: A Propensity Score Matching Analysis. J Gastrointest Surg (2018) 22(12):204554. doi: 10.1007/s11605-018-3854-2

30. Salvans S, Mayol X, Alonso S, Messeguer R, Pascual M, Mojal S, et al. Postoperative Peritoneal Infection Enhances Migration and Invasion Capacities of Tumor Cells In Vitro: An Insight Into the Association Between Anastomotic Leak and Recurrence After Surgery for Colorectal Cancer. Ann Surg (2014) 260(5):939-43; discussion 943-4. doi: 10.1097/ SLA.0000000000000958

31. Ueda K, Murakami J, Tanaka T, Nakamura T, Yoshimine S, Hamano K. Postoperative Complications and Cancer Recurrence: Impact on Poor Prognosis of Lower Lobe Cancer. Ann Thorac Surg (2020) 109(6):1750-6. doi: 10.1016/j.athoracsur.2019.12.061

32. Alonso S, Pascual M, Salvans S, Mayol X, Mojal S, Gil MJ, et al. Postoperative Intra-Abdominal Infection and Colorectal Cancer Recurrence: A Prospective Matched Cohort Study of Inflammatory and Angiogenic Responses as Mechanisms Involved in This Association. Eur J Surg Oncol (2015) 41 (2):208-14. doi: 10.1016/j.ejso.2014.10.052

33. Gowing SD, Cool-Lartigue JJ, Spicer JD, Seely AJE, Ferri LE. Toll-Like Receptors: Exploring Their Potential Connection With Post-Operative Infectious Complications and Cancer Recurrence. Clin Exp Metastases (2020) 37(2):225-39. doi: 10.1007/s10585-020-10018-2

34. Wu C, Wang N, Zhou H, Wang T, Mao Q, Zhang X, et al. Effects of Neoadjuvant Chemotherapy Toxicity and Postoperative Complications on Short-Term and Long-Term Outcomes After Curative Resection of Gastric Cancer. J Gastrointest Surg (2020) 24(6):1278-89. doi: 10.1007/s11605-019-04257-2

35. Perego M, Tyurin VA, Tyurina YY, Yellets J, Nacarelli T, Lin C, et al. Reactivation of Dormant Tumor Cells by Modified Lipids Derived From Stress-Activated Neutrophils. Sci Transl Med (2020) 12(572):eabb5817. doi: 10.1126/scitranslmed.abb5817

36. Jin LX, Sanford DE, Squires MH3rd, Moses LE, Yan Y, Poultsides GA, et al. Interaction of Postoperative Morbidity and Receipt of Adjuvant Therapy on Long-Term Survival After Resection for Gastric Adenocarcinoma: Results From the U.S. Gastric Cancer Collaborative. Ann Surg Oncol (2016) 23 (8):2398-408. doi: 10.1245/s10434-016-5121-7

37. Merkow RP, Bentrem DJ, Mulcahy MF, Chung JW, Abbott DE, Kmiecik TE, et al. Effect of Postoperative Complications on Adjuvant Chemotherapy Use for Stage III Colon Cancer. Ann Surg (2013) 258(6):847-53. doi: 10.1097/ SLA.0000000000000312

38. Pretzsch E, Bösch F, Renz B, Werner J, Angele M, Chaudry IH. Operative Trauma and Blood Loss - Impact on Tumor Growth and Recurrence. Shock (2021) 55(4):455-64. doi: 10.1097/SHK.0000000000001656

39. Wrba L, Palmer A, Braun CK, Huber-Lang M. Evaluation of Gut-Blood Barrier Dysfunction in Various Models of Trauma, Hemorrhagic Shock, and
Burn Injury. J Trauma Acute Care Surg (2017) 83(5):944-53. doi: 10.1097/ TA.0000000000001654

40. Yang J, Zhang J, Zhao C, Gai Z, Mu X, Wang Y, et al. Blood Loss Leads to Increase in Relative Abundance of Opportunistic Pathogens in the Gut Microbiome of Rabbits. Curr Microbiol (2020) 77(3):415-24. doi: 10.1007/ s00284-019-01825-2

41. Gruttadauria S, Saint Georges Chaumet M, Pagano D, Marsh JW, Bartoccelli C, Cintorino D, et al. Impact of Blood Transfusion on Early Outcome of Liver Resection for Colorectal Hepatic Metastases. J Surg Oncol (2011) 103(2):1407. doi: $10.1002 /$ jso. 21796

42. Su SF, Nieh HC. Efficacy of Forced-Air Warming for Preventing Perioperative Hypothermia and Related Complications in Patients Undergoing Laparoscopic Surgery: A Randomized Controlled Trial. Int J Nurs Pract (2018) 24(5):e12660. doi: 10.1111/ijn.12660

43. Pu Y, Cen G, Sun J, Gong J, Zhang Y, Zhang M, et al. Warming With an Underbody Warming System Reduces Intraoperative Hypothermia in Patients Undergoing Laparoscopic Gastrointestinal Surgery: A Randomized Controlled Study. Int J Nurs Stud (2014) 51(2):181-9. doi: 10.1016/ j.ijnurstu.2013.05.013

44. Wei Z, Tan B, Cao S, Liu S, Tan X, Yao Z, et al. The Influence of Neoadjuvant Chemotherapy on Gastric Cancer Patients' Postoperative Infectious Complications: What is the Negative Role Played by the Intestinal Barrier Dysfunction? Oncotarget (2017) 8(26):43376-88. doi: 10.18632/ oncotarget.14758

45. Gosavi R, Chia C, Michael M, Heriot AG, Warrier SK, Kong JC. Neoadjuvant Chemotherapy in Locally Advanced Colon Cancer: A Systematic Review and Meta-Analysis. Int J Colorectal Dis (2021) 36(10):2063-70. doi: 10.1007/ s00384-021-03945-3

46. Scilletta R, Pagano D, Spada M, Mongiovì S, Pesce A, Portale TR, et al. Comparative Analysis of the Incidence of Surgical Site Infections in Patients With Liver Resection for Colorectal Hepatic Metastases After Neoadjuvant Chemotherapy. J Surg Res (2014) 188(1):183-9. doi: 10.1016/j.jss.2013. 11.1092

47. Zhang Y, Ge L, Weng J, Tuo WY, Liu B, Ma SX, et al. Neoadjuvant Chemotherapy for Patients With Resectable Colorectal Cancer Liver Metastases: A Systematic Review and Meta-Analysis. World J Clin Cases (2021) 9(22):6357-79. doi: 10.12998/wjcc.v9.i22.6357

Conflict of Interest: The authors declare that the research was conducted in the absence of any commercial or financial relationships that could be construed as a potential conflict of interest.

Publisher's Note: All claims expressed in this article are solely those of the authors and do not necessarily represent those of their affiliated organizations, or those of the publisher, the editors and the reviewers. Any product that may be evaluated in this article, or claim that may be made by its manufacturer, is not guaranteed or endorsed by the publisher.

Copyright () 2022 Chen, Deng, Chen, Zhao, Bi, Zhou, Li, Huang, Zhang, Chen, Zhao and Cai. This is an open-access article distributed under the terms of the Creative Commons Attribution License (CC BY). The use, distribution or reproduction in other forums is permitted, provided the original author(s) and the copyright owner(s) are credited and that the original publication in this journal is cited, in accordance with accepted academic practice. No use, distribution or reproduction is permitted which does not comply with these terms. 